\title{
Sequências aritméticas de ordem superior e aplicações
}

\section{Higher order arithmetic sequences and applications}

DOI: $10.46814 / 1 a j d v 3 n 4-033$

Recebimento dos originais: 01/05/2021

Aceitação para publicação: 31/06/2021

Jhon Kenedy Moura Chagas

Mestrado em Agronomia pela Universidade de Brasília

Universidade de Brasília, Faculdade de Agronomia e Medicina Veterinária,

Campus Darcy Ribeiro, Brasília, Brasil.

E-mail: jhon.chagas@aluno.unb.br

\section{Josimar da Silva Rocha}

Doutorado em Matemática pela Universidade de Brasília, Universidade Tecnológica Federal do Paraná, Departamento Acadêmico de Matemática, Avenida Alberto Carazzai, 1640, Centro, Cornélio Procópio, Paraná, Brasil.

E-mail: jrocha@utfpr.edu.br

\section{RESUMO}

No cotidiano é comum lidarmos com algumas situações que envolvam a necessidade da determinação de uma regra geral para descrevê-las. Para ajudar na descrição de fenômenos que envolvam quantidades que podem ser expressas em termos de funções polinomiais surge a necessidade de encontrarmos instrumentos que facilitam a obtenção de tais funções polinomiais a partir dos dados analisados no processo. Uma maneira fácil de obtermos funções polinomiais a partir de uma sequência de números reais é por meio do estudo de Sequências Aritméticas de Ordem Superior. Se a sequência obtida a partir dos dados coletados por meio de um experimento for polinomial, então esta sequência será chamada de sequência aritmética de ordem $\mathrm{k}$, onde $\mathrm{k}$ é o grau do polinômio. Com o estudo de sequências aritméticas de ordem superior é possível obtermos a partir de uma sequência de números reais tanto a fórmula que descreve esta sequência quanto a fórmula da soma dos n primeiros termos desta sequência. Com isto, poderemos obter fórmulas gerais que nos permite obter uma estimativa para o comportamento de diversos fenômenos. Sendo assim, por meio do levantamento bibliográfico, consultando a referência [1] se deu início ao projeto de pesquisa com reuniões semanais.

Palavras-chave: Sequências. Polinômios. Triângulo de Pascal. Binômio de Newton. Funções.

\begin{abstract}
In everyday life it is common to deal with some situations that involve the need to determine a general rule to describe them. To help in the description of phenomena that involve quantities that can be expressed in terms of polynomial functions arises the need to find tools that facilitate the obtaining of such polynomial functions from the data analyzed in the process. An easy way to obtain polynomial functions from a sequence of real numbers is through the study of Higher Order Arithmetic Sequences. If the sequence obtained from the data collected through an experiment is polynomial, then this sequence will be called an arithmetic sequence of order $\mathrm{k}$, where $\mathrm{k}$ is the degree of the polynomial. With the study of higher order arithmetic sequences it is possible to obtain from a sequence of real numbers both the formula that describes this sequence and the formula for the sum of the first $\mathrm{n}$ terms of this sequence. With this, we can obtain general formulas that allow us to obtain an estimate for the
\end{abstract}


behavior of various phenomena. Thus, through a bibliographical survey, consulting reference [1], the research project began with weekly meetings.

Keywords: Sequences. Polynomials. Pascal's Triangle. Newton's binomial. Functions.

\section{INTRODUÇÃO}

Uma sequência aritmética de ordem superior é uma sequência em que a fórmula que a define é uma função polinomial. Desta forma, dizemos que uma sequência de ordem n é uma função polinomial de grau $n$.

Com o estudo de sequências aritméticas de ordem superior é possível obtermos a partir de uma sequência de números reais tanto a fórmula que descreve a sequência quanto a fórmula da soma dos $\mathrm{n}$ primeiros termos desta sequência. Assim, poderemos obter fórmulas gerais que nos permite obter uma estimativa para o comportamento de diversos fenômenos. Para mais informações veja [3].

\section{OBJETIVOS}

Como objetivos da pesquisa se teve o anseio de:

- Introduzir o conceito de sequência aritmética de ordem superior;

- Encontrar propriedades das sequências aritméticas de ordem superior;

- Buscar exemplos que ilustrem e justifiquem o estudo e a aplicação de sequências aritméticas de ordem superior.

\section{METODOLOGIA}

Inicialmente procurou-se estudar diversos conteúdos que serviram de prérequisitos para o estudo de sequências aritméticas, tais como progressões aritméticas, triângulo de Pascal, binômio de Newton, entre outros. Para tal foi utilizada basicamente a referência [1].

Através do estudo introdutório surgiu a necessidade do estudo de técnicas matemáticas de indução para a obtenção de resultados gerais que foram contemplados nas demonstrações dos principais resultados. Este foi possível pela consulta a [4].

Foram necessárias pesquisas bibliográficas, utilizamos, por exemplo, [3], para a obtenção de aplicações envolvendo sequências aritméticas de ordem superior por meio de fenômenos cujo comportamento pode ser traduzido através de uma função polinomial.

Um estudo de programação na linguagem $C$ utilizando [2] também foi necessário para o desenvolvimento de um software para integrar parte dos conhecimentos sobre progressões aritméticas de ordem superior, sendo que, para alcançar este fim, consultamos [6]. Uma versão deste programa pode ser obtido no apêndice deste trabalho. Na figura 1 temos uma captura de tela da execução do programa. 
Figura 1 - Exemplo de utilização do programa desenvolvido

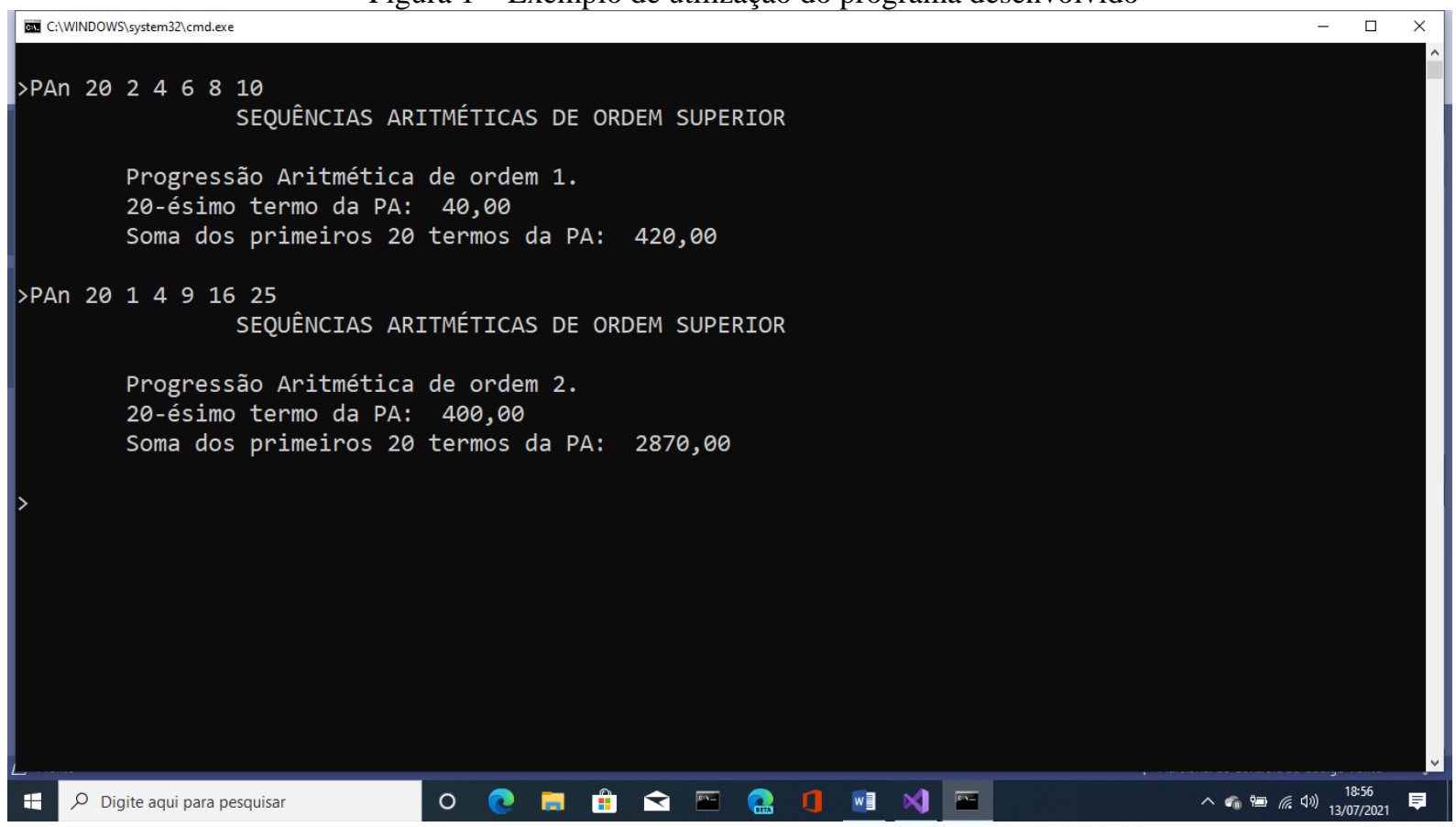

\section{RESULTADOS E DISCUSSÃO}

Primeiramente criamos uma notação especial para designar cada termo da tabela das diferenças. Em $a_{n}{ }^{(c)}$, de acordo com a tabela das diferenças, (c) indica em qual linha se encontra o termo e $\mathrm{n}$ indica a posição do termo na linha indicada. Desde então esta foi utilizada para se referir aos termos das sequências utilizadas no contexto da tabela das diferenças.

Logo após definimos o que chamamos de tabela das diferenças. Esta será usada para obter a partir da sequência $\left(a_{n}{ }^{(1)}\right)$ a diferença entre seus termos que será tida como uma outra sequência, $\left(a_{n}{ }^{(2)}\right)$, fazendo isto por $k+1$ vezes para obter a sequência $\left(a_{n}{ }^{(k+1)}\right)$. Dessa forma o termo $a_{n}{ }^{(c)}$ será obtido recursivamente pela equação (1).

$$
a_{n}^{(c)}=a_{n+1}^{(c-1)}-a_{n}^{(c-1)}
$$

Logo, uma sequência numérica $\left(a_{n}\right)$ é uma sequência aritmética de ordem $\mathrm{k}$ se a sequência $\left(\mathrm{a}_{\mathrm{n}}{ }^{(\mathrm{k}+1)}\right)$ obtida a partir da tabela das diferenças é constante para alguma constante diferente de zero.

Por meio de discussões chegamos à determinação de uma fórmula para o termo geral de uma sequência aritmética de ordem k. Esta é expressa segundo a equação (2).

$$
a_{n}=\sum_{j=0}^{k}\left(\begin{array}{c}
n-1 \\
j
\end{array}\right) a_{1}^{(j+1)}
$$


Utilizando as propriedades vistas em [1] e [5] conseguimos demonstrar uma fórmula que descreve o comportamento da soma dos $\mathrm{n}$ primeiros termos de uma sequência aritmética de ordem $\mathrm{k}$. A equação 3 representa a fórmula da soma.

$$
S_{n}=\sum_{j=0}^{k}\left(\begin{array}{c}
n \\
j+1
\end{array}\right) a_{1}^{(j+1)}
$$

Para calcular os coeficientes binomiais presentes até então nas equações (2) e (3) utilizamos a equação (4), sendo que esta equação é válida para todo n e p naturais.

$$
\left(\begin{array}{l}
n \\
p
\end{array}\right)=\frac{n \cdot(n-1) \cdot(n-2) \cdot \ldots \cdot(n-(p-1))}{p !}
$$

Após conseguirmos obter fórmulas gerais para $a_{n}$ e $S_{n}$ partimos para o estudo de casos que nos conduziram a uma diversidade de pontos que poderiam ser desenvolvidos durante a pesquisa.

Percebemos que a soma de qualquer linha da tabela das diferenças, a partir da segunda, com o primeiro elemento da linha anterior resulta no termo $a_{n+1}{ }^{(1)}$ conforme descrito na equação 5 .

$$
S_{n}^{(2)}+a_{1}^{(1)}=a_{n+1(5)}^{(1)}
$$

Tendo uma sequência $\left(a_{1}, a_{2}, \ldots, a_{n}\right)$ como uma sequência aritmética de ordem $\mathrm{k}$ podemos afirmar que a sequência inversa $\left(\mathrm{a}_{\mathrm{m}}, \mathrm{a}_{\mathrm{m}-1}, \ldots, \mathrm{a}_{1}\right)$ também é uma sequência aritmética de mesma ordem.

A partir do que foi referido podemos afirmar que permutando os n elementos de uma sequência qualquer, desde que esta seja uma sequência aritmética de ordem k, há no mínimo mais uma sequência que é uma sequência aritmética de ordem k. Podendo existir mais outras de mesma ordem ou não. Para esse fenômeno não pudemos estabelecer regras gerais que expliquem esse comportamento. Apenas analisamos alguns exemplos.

Para garantir que a partir da tabela das diferenças determinada sequência seja uma sequência aritmética de ordem $\mathrm{k}$ necessitamos apenas de $\mathrm{k}+1$ termos.

Sendo que caso se utilize mais ou menos termos a sequência pode ou não ser uma sequência aritmética de ordem superior com ordem maior ou menor que $\mathrm{k}$. 
Enfim, outras propriedades e aspectos de menor relevância também foram estudados durante a pesquisa. De modo que os supracitados podem ser vistos como os de principal importância.

\section{CONCLUSÕES}

Além dos resultados que já esperávamos obter, que se referem ao termo geral e à fórmula da soma dos n primeiros termos, conseguimos obter conclusões que evidenciam a existência de sequências aritméticas de ordem superior e nos conduzem a perceber sua aplicação em diversos problemas.

As equações (2) e (3) foram demonstradas pelo estudo das propriedades descritas em [5] e pelas técnicas de indução apresentadas em [4]. A equação (2), a fórmula do termo geral de uma sequência aritmética de ordem superior, pode ser utilizada tanto para descobrir qualquer termo n da sequência ou o polinômio que descreve esta sequência. Assim, uma conclusão que pode ser obtida foi que o polinômio possui o mesmo grau da sequência que ele descreve, ou seja, ambos possuem grau k.

Já quando nos referimos a equação (3), a fórmula da soma dos $\mathrm{n}$ primeiros termos de uma sequência aritmética de ordem superior, podemos concluir que o grau do polinômio que a gera é um grau maior que o da sequência, isto é, a sequência possui grau k e o polinômio da soma possui grau $\mathrm{k}+1$.

Não conseguimos demonstrar e obter casos gerais para alguns fenômenos, o que abre margem para que outros pesquisadores possam continuar a desenvolver essa temática e estabelecer regras e explicações para o comportamento desses fenômenos. 


\section{Referências}

[1] IEZZI, Gelson et al. Matemática - Volume Único. São Paulo: Atual, 2007.

[2] KLANDRES, Lars; KRIS, Jamsa. Programando em C/C++: "A Bíblia". São Paulo: Makron Books do Brasil Ltda, 1999.

[3] LOPES, Luís. Manual de Progressões. Rio de Janeiro: Interciência, 1998.

[4] MORGADO, José. Indução e Indução Matemática. Boletim da SPM nº17 de junho de 1990. Disponível em: 〈http://nautilus.fis.uc.pt/bspm/revistas/17/023034.150.pdf>. Acesso em: 07 nov. de 2011.

[5] RODRIGUES, F. W. O Binômio de Newton e a Vênus de Milo. RPM 30, SBM, 1996, págs. 34-41.

[6] RUGGIERO, Márcia A. Gomes; LOPES, Vera Lúcia da Rocha. Cálculo Numérico: Aspectos Teóricos e Computacionais. São Paulo: McGraw-Hill, 1998. 


\section{APÊNDICE}

\#include<stdio.h>

\#include<stdlib.h>

\#include<locale.h>

// Este programa deve receber uma lista de parãmetros numéricos (n, x, y, z, ..., w)

// sendo $\mathrm{n}$ um número inteiro e $(\mathrm{x}, \mathrm{y}, \mathrm{z}, \ldots, \mathrm{w})$ os temos de uma $\mathrm{PA}$,

// imprimindo a ordem da Progressão Aritmética, o n-ésimo termo e a soma dos

// n primeiros termos da Progressão Aritmética.

//Função que calcula um número binomial:

double binomio(int numero1, int numero2) \{

if (numero2 > numero1) return 0 ;

else if (numero2 ==1) return numero1;

else if (numero2 $==0$ ) return 1 ;

else if (numero2 == numero1) return 1;

else return binomio(numero1 - 1, numero2) + binomio(numero1 - 1, numero 2 - 1);

\}

//Função que calcula os elementos da tabela das diferenças:

double seq(double * vetor, int linha, int coluna) \{

if (linha $=0$ ) return vetor[coluna];

else return seq(vetor, linha -1 , coluna +1 ) - seq(vetor, linha - 1, coluna);

\}

//Função que encontra a ordem da Progressão Aritmética:

int ordem(double* vetor, int dim) \{

int $\mathrm{i}$;

for $(\mathrm{i}=0 ; \mathrm{i}<\operatorname{dim} ; \mathrm{i}++)$

if (seq(vetor, dim - i, 0) !=0) break;

return dim - i; 
//Função que calcula o n-ésimo termo de uma Progressão Aritmética:

double termo(double* vetor, int dim, int numero) \{

double $\mathrm{x}=0$;

int i;

$$
\begin{aligned}
& \text { for }(\mathrm{i}=0 ; \mathrm{i}<=\operatorname{dim} ; \mathrm{i}++) \\
& \qquad \mathrm{x}=\mathrm{x}+\operatorname{binomio}(\text { numero }-1, \mathrm{i}) * \operatorname{seq}(\text { vetor, } \mathrm{i}, 0) ;
\end{aligned}
$$

return $\mathrm{x}$;

\}

// Função que determina a soma dos k primeiros termos de uma Progressão Aritmética:

double soma(double * vetor, int dim, int numero) \{

double $\mathrm{x}=0$;

int $\mathrm{i}$;

for $(\mathrm{i}=0 ; \mathrm{i}<=\operatorname{dim} ; \mathrm{i}++)$

$$
\mathrm{x}=\mathrm{x}+\operatorname{binomio}(\text { numero }, \mathrm{i}+1) * \operatorname{seq}(\text { vetor }, \mathrm{i}, 0) \text {; }
$$

return $\mathrm{x}$;

\}

//Programa principal:

int main(int argc, char* argv[]) \{

//Configuração dos parâmetros locais de Linguagem e acentuação:

setlocale(LC_ALL, "Portuguese");

//Para que o programa continue o usuário deve digitar pelo menos dois números como parâmetros:

$$
\begin{aligned}
& \text { if }(\operatorname{argc}>2)\{ \\
& \text { double* vetor; } \\
& \text { int } \mathrm{i} ;
\end{aligned}
$$

//Alocação dinâmica de memória para os termos da Progressão2 Aritmética:

vetor $=($ double $*)$ malloc $((\operatorname{argc}-1) * \operatorname{sizeof}($ double $))$;

//Preenchimento de um vetor com os termos da Progressão Aritmética:

$$
\begin{aligned}
\text { for }(\mathrm{i}= & 0 ; \mathrm{i}<\operatorname{argc}-2 ; \mathrm{i}++) \\
& \operatorname{vetor}[\mathrm{i}]=\operatorname{atof}(\operatorname{argv}[\mathrm{i}+2]) ;
\end{aligned}
$$


//Impressão dos resultados:

$\mathrm{i}=\operatorname{atoi}(\operatorname{argv}[1])$;

printf("\t|tSEQUÊNCIAS ARITMÉTICAS DE ORDEM SUPERIOR\n\n");

printf("\tProgressão Aritmética de ordem \%d.ın", ordem(vetor, $\operatorname{argc}$ - 3));

printf("\t\%d-ésimo termo da PA: \%.2fln", i, termo(vetor, $\operatorname{argc}-3, \mathrm{i})$ );

printf("\tSoma dos primeiros \%d termos da PA: \%.2fln", i, soma(vetor, $\operatorname{argc}-3, \mathrm{i})$ );

//Liberação da memória alocada dinamicamente:

free(vetor);

\}

else printf("Digite pelo menos dois parâmetros numéricos!!n");

return 0 ; 